\title{
Achieving Stability and Fairness in Mobile Ad Hoc Networks ${ }^{1}$
}

\author{
Ahmed M. Mahdy, Jitender S. Deogun, and Jun Wang \\ Computer Science and Engineering Department, \\ University of Nebraska-Lincoln, Lincoln, \\ NE 68588 USA \\ \{amahdy, deogun, wang\}@cse.unl.edu
}

\begin{abstract}
In this paper, we present an approach to stable and fair mobile ad hoc networks. Our approach targets desired quality features using customizable clustering algorithms. Simulation results show that significant improvement on network stability and fairness can be achieved using our approach.
\end{abstract}

\section{Introduction}

The infrastructure organization of mobile ad hoc networks has received great attention in the last several years. The widely used cluster-based scheme organizes the network into a layered hierarchy. In this organization, the higher the layer that a node belongs to, the more responsibilities a node carries. This architecture was first used in hierarchal routing in [1]. The two layer (i.e. clusterhead based) scheme is the most popular among the layered hierarchal schemes due to its relative simplicity and less overhead compared to schemes with more than two layers. In clusterhead based networks, the nodes are grouped into clusters supervised by clusterheads. Two major issues are defined in this area; cluster formation and clusterhead selection. Cluster formation refers to how the network is divided into clusters. Static and dynamic clustering are the two cluster formation methods discussed in the literature. Static clustering predetermines the clusters shape and size similar to cellular networks. In contrary, dynamic clustering has no fixed organization and depends on the behavior of the nodes. On the other hand, the research on the selection of clusterheads focuses on the development of selection criteria that distinguish the nodes according to some quality measures such as connectivity degree and mobility.

The effect of the clustering techniques on the network performance is commonly evaluated in terms of network stability and fairness (i.e. load balance). Network stability is adversely proportional to the number of clusterhead replacements; the less the number of clusterhead changes is, the more stable the network is. On the other hand, an ideally fair clustering technique uniformly distributes the managerial load over network nodes. The more the number of nodes involved in the management of the

\footnotetext{
${ }^{1}$ This work was supported, in part, by an NSF EPSCoR grant EPS-0346476 and by a Nebraska Research Initiative grant on high performance wireless networks.
} 
network is, the fairer the technique is. There is a tradeoff between network stability and fairness. One of the two merits is sacrificed on the account of the other in many cases for the sake of simpler designs. This can significantly deteriorate network performance. Therefore, the clustering technique should be able to strike a tradeoff between stability and fairness in order to achieve better overall performance. We believe that clustering techniques should be adaptable and configurable to seek specific network merits. The same technique should be configurable for maximum stability, maximum fairness, or optimized overall performance

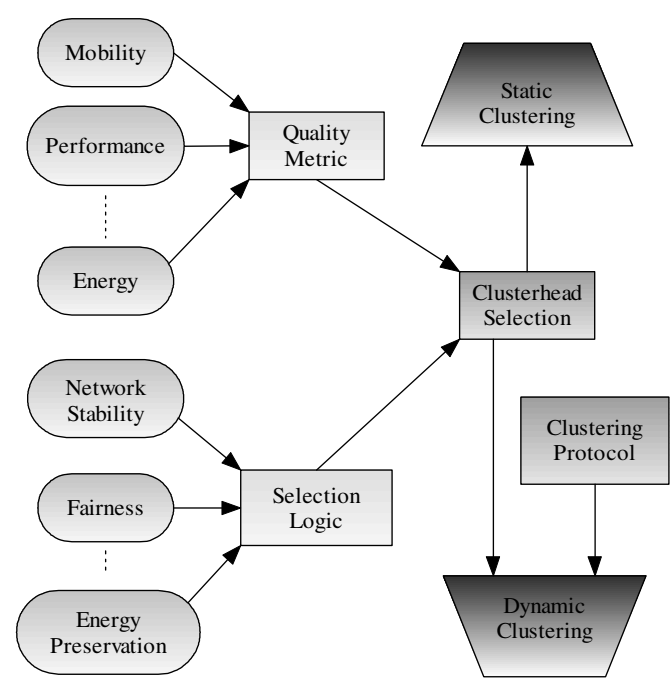

Fig. 1. A block diagram of the clustering framework

\section{Stable and Fair Clustering}

The management of clusterhead schemes is carried out through a two-step process; cluster formation and clusterhead selection. A clustering protocol regulates cluster formation and coordinates the communication between various nodes. Once the clusters are formed, clusterheads are selected according to some selection criterion.

We propose a clustering framework that generates customizable clustering methods with multi-quality measure selection criteria. The selection logic subcomponent of our framework controls the selection behavior through which the desirable network merits can be achieved. Technically, it is responsible for determining when a new clusterhead should be selected. The number of selection rounds and the frequency of clusterhead replacements greatly affect network stability and fairness. They depend on the logic of the selection algorithm. Accordingly, this subcomponent comprises different selection algorithms that are associated with specific network merits. The desired network merits, therefore, determine which selection algorithm should be used. 


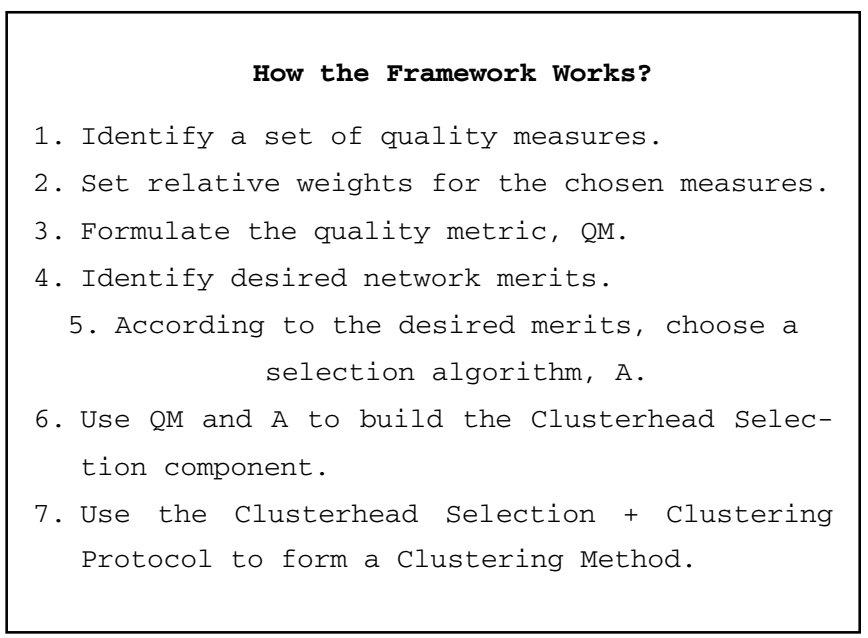

Fig. 2. How the framework works

Ideally, the outcome of the selection process should improve the performance of the cluster as well as the network. Therefore, we evaluate the performance of a selection algorithm based on our framework against the WCA algorithm [2]. Our algorithm is composed of two components, namely Control Loop (CL) and Event Triggered (ET). We designed our algorithm so that it can cover all three possible combinations of stability and fairness by customizing the behavior of the CL and ET components. The CL component focuses on network fairness more than network stability; in contrast the ET component is responsible of achieving high network stability. Our algorithm is designed so that it compromises the tradeoff between the two merits by seeking a midpoint performance.

\section{Performance Evaluation}

We evaluate the network performance using the following metrics. Number of Switches (NS) is the number of times a clusterhead is replaced. It is an indication of the amount of overhead the system has to afford and a sign of how stable the system is. Maximum Service time Percentage (MSP) is the maximum time a node spends as a clusterhead as a percentage of the total time; a sign of load balance. Energy Standard Deviation (ESD) is the standard deviation of the energy level at the end of the simulation. The results are normalized to the best case scenario where all nodes consume the same amount of energy. This metric reflects the effect of the selection process on the energy consumption and is a sign of system fairness. The larger this measure is, the less fair the system is. We simulate the algorithms performance on a mobile ad hoc network of size 100 to 500 nodes. The results show that our algorithm outperforms WCA achieving improvement of up to $10 \%, 58 \%$, and $28 \%$ on the NS, MSP, and ESD, respectively. 

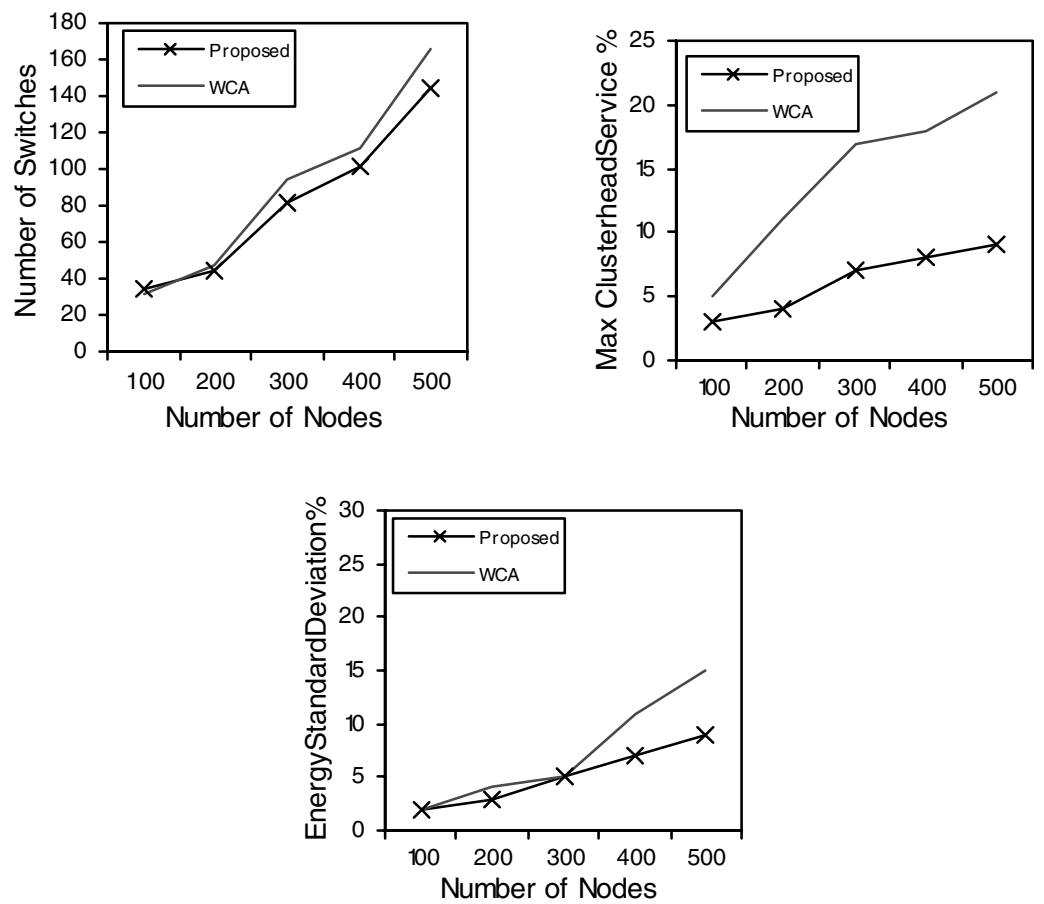

Fig. 2. Performance of the proposed algorithm against the WCA algorithm

\section{References}

1. Ephremides, A., Wieselthier, J.E., and Baker, D.J.: A Design Concept for Reliable Mobile Radio Networks with Frequency Hopping Signaling. Proc. of the IEEE, 1(1987) 56-73

2. Chatterjee, M., Das, S.K., and Turgut, D.: WCA: A Weighted Clustering Algorithm for Mobile Ad hoc Networks. Journal of Clustering Computing, 2(2002) 193-204 